\title{
De Sitter vacua in no-scale supergravity
}

\author{
John Ellis, ${ }^{a, b, c}$ Balakrishnan Nagaraj, ${ }^{d}$ Dimitri V. Nanopoulos ${ }^{d, e, f}$ and Keith A. Olive ${ }^{g}$ \\ ${ }^{a}$ Theoretical Particle Physics and Cosmology Group, Department of Physics, \\ King's College London, London WC2R 2LS, U.K. \\ ${ }^{b}$ Theoretical Physics Department, CERN, \\ CH-1211 Geneva 23, Switzerland \\ ${ }^{c}$ National Institute of Chemical Physics 6 Biophysics, \\ Rävala 10, 10143 Tallinn, Estonia \\ ${ }^{d}$ George P. and Cynthia W. Mitchell Institute for Fundamental Physics and Astronomy, \\ Texas A $6 M$ University, College Station, TX 7r843, U.S.A. \\ ${ }^{e}$ Astroparticle Physics Group, Houston Advanced Research Center (HARC), \\ Mitchell Campus, Woodlands, TX 77381, U.S.A. \\ ${ }^{f}$ Academy of Athens, Division of Natural Sciences, \\ Athens 10679, Greece \\ ${ }^{g}$ William I. Fine Theoretical Physics Institute, School of Physics and Astronomy, \\ University of Minnesota, Minneapolis, MN 55455, U.S.A. \\ E-mail: John.Ellis@cern.ch, nbala@physics.tamu.edu, \\ dimitri@physics.tamu.edu, olive@umn.edu
}

ABSTRACT: No-scale supergravity is the appropriate general framework for low-energy effective field theories derived from string theory. The simplest no-scale Kähler potential with a single chiral field corresponds to a compactification to flat Minkowski space with a single volume modulus, but generalizations to single-field no-scale models with de Sitter vacua are also known. In this paper we generalize these de Sitter constructions to twoand multi-field models of the types occurring in string compactifications with more than one relevant modulus. We discuss the conditions for stability of the de Sitter solutions and holomorphy of the superpotential, and give examples whose superpotential contains only integer powers of the chiral fields.

KEYWORDS: Supergravity Models, Superstring Vacua

ARXIV EPRINT: 1809.10114 


\section{Contents}

1 Introduction 1

2 Single-field models 2

2.1 No-scale supergravity models 2

2.2 Minkowski solutions 4

2.3 De Sitter solutions 5

$3 \quad$ Two-field models $\quad 6$

3.1 Minkowski solutions 6

$\begin{array}{llr}3.2 & \text { De Sitter solutions } & 10\end{array}$

3.3 Stability analysis 11

$4 \quad$ N-field models $\quad 13$

4.1 Minkowski Solutions 13

$\begin{array}{lll}4.2 & \text { De Sitter solutions } & 15\end{array}$

$\begin{array}{lll}4.3 & \text { Stability analysis } & 16\end{array}$

5 Conclusion and outlook $\quad 18$

\section{Introduction}

If one assumes that $N=1$ supersymmetry holds down to energies hierarchically smaller than the Planck mass, low-energy dynamics must be governed by some $N=1$ supergravity. It is known that the energy density in the present vacuum is very small compared, e.g., to typical energy scales in the Standard Model. It was therefore natural to look for $N=1$ supergravity theories that yielded a vanishing cosmological constant without unnatural fine tuning, and a total scalar potential that is positive definite. The unique Kähler potential for such an $N=1$ supergravity model with a single chiral superfield $\phi$ (up to canonical field redefinitions) was found in [1] to be

$$
K=-3 \ln \left(\phi+\phi^{\dagger}\right) .
$$

In [2] this was dubbed 'no-scale supergravity', because the scale of supersymmetry breaking is undetermined at the tree level, and it was suggested that the scale might be set by perturbative corrections to the effective low-energy field theory. The single-field model (1.1) was explored in more detail in [3] (EKN), and the generalization to more superfields was developed in [4]. ${ }^{1}$ It was shown subsequently that no-scale supergravity emerges as the

\footnotetext{
${ }^{1}$ For a review of early work on no-scale supergravity, see [5].
} 
effective field theory resulting from a supersymmetry-preserving compactification of tendimensional supergravity, used as a proxy for compactification of heterotic string theory [6].

In recent years interest has grown in the possibility of string solutions in de Sitter space, for at least a couple of practical reasons. One is the discovery that the expansion of the Universe is accelerating due to non-vanishing vacuum energy that is small relative to the energy scale of the Standard Model [7, 8] (for the most recent observational constraints see [9]). The other is the growing observational support for inflationary cosmology [10-16], according to which the Universe underwent an early epoch of near-exponential quasi-de Sitter expansion driven by vacuum energy that was large compared with the energy scale of the Standard Model, but still hierarchically smaller than the Planck scale. At the time of writing there is an ongoing controversy whether string theory in fact admits consistent solutions in de Sitter space [17-29].

If string theory does indeed admit de Sitter solutions and approximate supersymmetry with scales hierarchically smaller than the string scale, their low-energy dynamics should be described by some suitable supergravity theory that is capable of incorporating the breaking of supersymmetry that is intrinsic in de Sitter space. Since string compactifications yield no-scale supergravity as an effective low-energy field theory, it is natural to investigate how de Sitter space could be accommodated within no-scale supergravity. ${ }^{2}$ This question was studied already in [3], and the purpose of this paper is to analyze this question in more detail and generality, extending the previous single-field analysis of $[3,36]$ to no-scale models with multiple superfields that are characteristic of generic string compactifications. These models may provide a useful guide to the possible forms of effective field theories describing the low-energy dynamics in de Sitter solutions of string theory, assuming that they exist.

The outline of this paper is as follows. In section 2 we review the original motivation and construction of no-scale supergravity with a vanishing cosmological constant [1], and also review the construction in $[3,36]$ of no-scale supergravity models with non-vanishing vacuum energy. Section 3 describes the extensions of these models to no-scale supergravity models with two chiral fields, which have an interesting geometrical visualization. The de Sitter construction is extended to multiple chiral fields in section 4. In each case, we discuss the requirements of stability of the vacuum and holomorphy of the superpotential, and give examples of models whose superpotentials contain only integer powers of the chiral fields. Finally, section 5 summarizes our conclusions and presents some thoughts for future work.

\section{Single-field models}

\section{$2.1 \quad$ No-scale supergravity models}

We recall that the geometry of a $N=1$ supergravity model is characterized by a Kähler potential $K$ that is a Hermitian function of the complex chiral fields $\phi^{i}$. The kinetic terms of these fields are

$$
K_{i}^{j} \frac{\partial \phi_{i}}{\partial x_{\mu}} \frac{\partial \phi_{j}^{\dagger}}{\partial x^{\mu}} \quad \text { where } \quad K_{i}^{j} \equiv \frac{\partial^{2} K}{\partial \phi^{i} \partial \phi_{j}^{\dagger}}
$$

\footnotetext{
${ }^{2}$ For other approaches, see [30-35].
} 
is the Kähler metric. Defining also $K_{i} \equiv \partial K / \partial \phi^{i}$ and analogously its complex conjugate $K^{i}$, the tree-level effective potential is

$$
V=e^{K}\left[K^{j} K_{i}^{-1 j} K_{i}-3\right]+\frac{1}{2} D^{a} D^{a},
$$

where $K_{i}^{-1 j}$ is the inverse of the Kähler metric (2.1) and $\frac{1}{2} D^{a} D^{a}$ is the $D$-term contribution, which is absent for chiral fields that are gauge singlets as we assume here.

In this section we consider the case of a single chiral field $\phi$, in which case it is easy to verify that the first term in (2.2) can be written in the form

$$
V(\phi)=9 e^{4 K / 3} K_{\phi \phi^{\dagger}}^{-1} \partial_{\phi} \partial_{\phi^{\dagger}} e^{-K / 3} .
$$

It is then clear that the unique form of $K$ with a Minkowski solution, for which $V=0$, is

$$
K=-3 \ln \left(f(\phi)+f^{\dagger}\left(\phi^{\dagger}\right)\right)
$$

where $f$ is an arbitrary analytic function. In fact, since physical results are unchanged by canonical field transformations, one can transform $f(\phi) \rightarrow \phi$ and recover the simple form (1.1) of the Kähler potential for a no-scale supergravity model with a single chiral field.

We note that this Kähler potential describes a maximally-symmetric $\mathrm{SU}(1,1) / \mathrm{U}(1)$ manifold whose Kähler curvature $R_{i}^{j} \equiv \partial_{i} \partial^{j} \ln K_{i}^{j}$ obeys the simple proportionality relation

$$
\frac{R_{i}^{j}}{K_{i}^{j}} \equiv R=\frac{2}{3},
$$

which is characteristic of an Einstein-Kähler manifold.

This model was generalized in EKN [3], where general solutions for all flat potentials were found. The $\mathrm{SU}(1,1)$ invariance in eq. (2.4) holds whenever ${ }^{3}$

$$
R \equiv \frac{R_{i}^{j}}{K_{i}^{j}}=\frac{2}{3 \alpha},
$$

which corresponds (up to irrelevant field redefinitions) to the extended Kähler potential

$$
G=K+\ln W(\phi)+\ln W^{\dagger}\left(\phi^{\dagger}\right),
$$

where

$$
K=-3 \alpha \ln \left(\phi+\phi^{\dagger}\right),
$$

we assume $\alpha>0$, and $W(\phi)$ is the superpotential. ${ }^{4}$ In this case the effective potential is

$$
V=e^{G}\left[G^{j} K_{i}^{-1 j} G_{i}-3\right] .
$$

\footnotetext{
${ }^{3}$ We note that in extended SU(N,1) no-scale models [4] that include $N-1$ matter fields, $y_{i}$, with the Kähler potential $K=-3 \alpha \log \left(\phi+\phi^{\dagger}-y^{i} y_{i}^{\dagger} / 3\right)$, the Kähler curvature becomes $R=(N+1) / 3 \alpha$. Our constructions can be generalized to this case, but such generalizations lie beyond the scope of this paper.

${ }^{4}$ Starobinsky-like models with $\alpha \neq 1$ were discussed in [37]. Such models were later dubbed $\alpha$-attractors in $[36,38,39]$.
} 
EKN found 3 classes of solutions with a constant scalar potential [3], namely

$$
\begin{aligned}
& \text { 1) } \quad W=a \quad \text { and } \quad \alpha=1 \\
& \text { 2) } \quad W=a \phi^{3 \alpha / 2}, \\
& \text { 3) } \quad W=a \phi^{3 \alpha / 2}\left(\phi^{3 \sqrt{\alpha} / 2}-\phi^{-3 \sqrt{\alpha} / 2}\right) .
\end{aligned}
$$

Solution 1) corresponds to the $V=0$ Minkowski solution discussed above, whereas solutions 2) and 3) yield potentials that are constant in the real direction, but are in unstable in the imaginary direction. As we discuss further below, stabilization in the imaginary direction is straightforward and allows these solutions to be used for realistic models with constant non-zero potentials in the real direction. We find that 2) leads to anti-de Sitter solutions with $V=-3 / 8^{\alpha} \cdot a^{2}$ and 3) leads to de Sitter solutions ${ }^{5}$ with $V=3 \cdot 2^{2-3 \alpha} \cdot a^{2}$. We note that in the particular case $\alpha=1$ this reduces to $W=a\left(\phi^{3}-1\right)$, which yields the de Sitter solution discussed in [36]. This was utilized in [40] when making the correspondence between no-scale supergravity and $R^{2}$ gravity.

In the following subsections, we first generalize the Minkowski solution (2.10), and then show that de Sitter solutions can be obtained as combinations of Minkowski solutions. These aspects of the solutions will subsequently be used to generalize them to model theories with multiple moduli.

\subsection{Minkowski solutions}

We consider the $N=1$ no-scale supergravity model with a single complex chiral field $\phi$ described by the Kähler potential given in (2.8) and the superpotential $W(\phi)$ is a monomial of the form

$$
W=a \phi^{n},
$$

and we seek the value of $n$ that admits a Minkowski solution with $V=0$. Defining $\phi \equiv x+i y$, the potential along real field direction $x$ is given by

$$
V=2^{-3 \alpha} \cdot\left(\frac{(2 n-3 \alpha)^{2}}{3 \alpha}-3\right) \cdot a^{2} \cdot x^{2 n-3 \alpha}
$$

We can obtain a Minkowski solution by setting to zero the term in the brackets:

$$
\frac{(2 n-3 \alpha)^{2}}{3 \alpha}=3
$$

Solving the above equation for $n$, we find two solutions [36]:

$$
n_{ \pm}=\frac{3}{2}(\alpha \pm \sqrt{\alpha})
$$

We note that $n_{-}=0$ for $\alpha=1$, corresponding to the EKN solution (2.10) listed above. However, we see that in addition to this $n=0$ solution, $n=3$ also yields a Minkowski solution with $V=0$ in all directions in field space.

\footnotetext{
${ }^{5}$ We correct here a typo in the third solution given in [3].
} 
Although such solutions exist for any $\alpha$, for the superpotential to be holomorphic we need $n_{-} \geq 0$, which requires $\alpha \geq 1$. Clearly, integer solutions for $n$ are obtained whenever $\alpha$ is a perfect square [36].

It is possible to go from one superpotential to another via a Kähler transformation:

$$
K \longrightarrow K+\lambda(\phi)+\lambda^{\dagger}\left(\phi^{\dagger}\right), \quad W \longrightarrow e^{-\lambda(\phi)} W .
$$

with $\lambda(\phi)= \pm 3 \sqrt{\alpha} \ln \phi$. In general, the solutions (2.16) can be thought of as corresponding to endpoints of a line segment of length $3 \sqrt{\alpha}$ centred at $3 \alpha / 2$. Though this appears trivial, extensions of this geometric visualization will be useful in the generalizations to multiple fields discussed below.

For $\alpha \neq 1$, the two solutions yield $V=0$ only along the real direction, and the mass squared of the imaginary component $y$ along the real field direction for $x>0$ and $y=0$ is given by

$$
m_{y}^{2}=2^{2-3 \alpha} \cdot \frac{(\alpha-1)}{\alpha} \cdot a^{2} \cdot x^{ \pm 3 \sqrt{\alpha}}
$$

where the \pm in the exponent corresponds to the two solutions $n_{ \pm}$. From this it is clear that the Minkowski solutions are stable for $\alpha \geq 1$.

There are two aspects of the single-field model that we emphasize here, because they generalize in an interesting way to multi-field models. The first is the fact that there are two solutions for $n$ and the second is that, when $\alpha=1$, we get a Minkowski solution with a potential that vanishes everywhere.

\subsection{De Sitter solutions}

As was shown in EKN, de Sitter solutions can be found with the Kähler potential (2.8) and a superpotential of the form (2.12), which may be written as

$$
W=a\left(\phi^{n_{-}}-\phi^{n_{+}}\right),
$$

where $n_{ \pm}$are given in (2.16). In this case the potential along the real field direction $y=0$ is

$$
V=3 \cdot 2^{2-3 \alpha} \cdot a^{2} .
$$

Thus, the de Sitter solution is obtained by taking the difference of the two "endpoint" solutions mentioned above.

Unfortunately, this de Sitter solution is not stable for finite $\alpha$. However, this can be remedied by deforming the Kähler potential to the following form [37, 41]:

$$
K=-3 \alpha \ln \left(\phi+\phi^{\dagger}+b\left(\phi-\phi^{\dagger}\right)^{4}\right): b>0 .
$$

The addition of the quartic stabilization term does not modify the potential in the real direction, which is still given by (2.20). However, the squared mass of the imaginary component $y$ is now given by

$$
m_{y}^{2}=\frac{2^{2-3 \alpha}}{\alpha} \cdot a^{2} \cdot x^{-3 \sqrt{\alpha}} \cdot\left(\alpha\left(x^{3 \sqrt{\alpha}}-1\right)^{2}-\left(1-96 b x^{3}\right)\left(x^{3 \sqrt{\alpha}}+1\right)^{2}\right) .
$$

The stability requirement $m_{y}^{2} \geq 0$ is achieved when $\alpha \geq 1$. In figure 1 we plot the stabilized potential for $a=b=\alpha=1$, and we see that the potential is completely flat along the line $y=0$ and is stable for all values of $x>0$. 


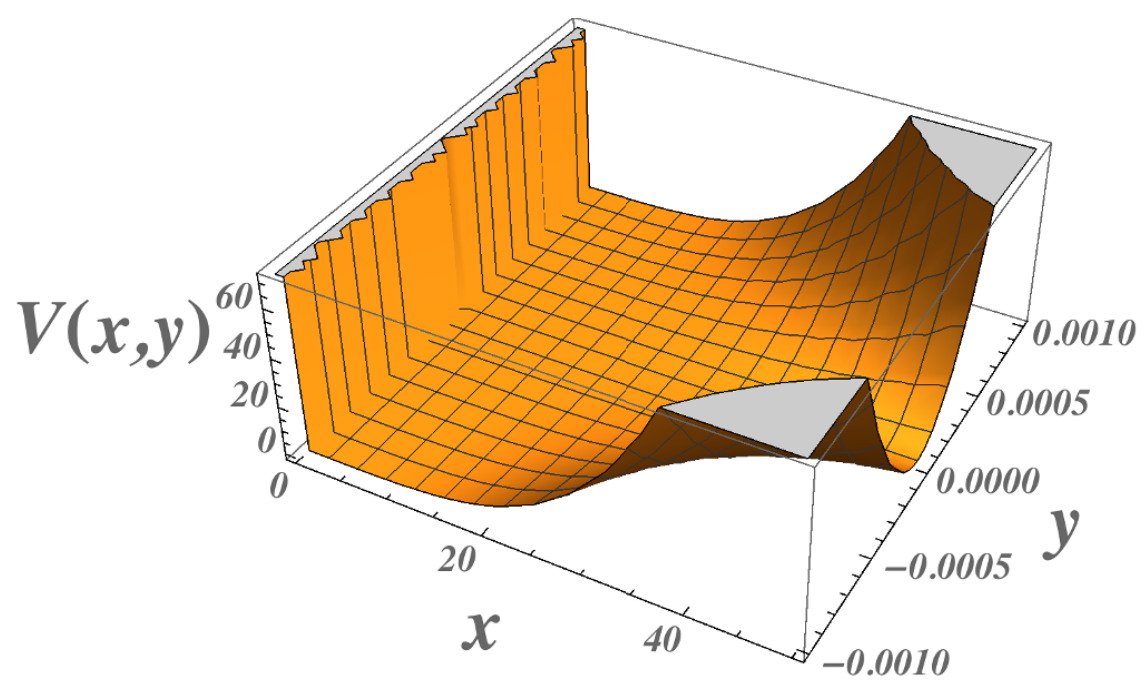

Figure 1. The potential $V(x, y)$ for $a=b=\alpha=1$ in no-scale supergravity with the stabilized Kähler potential (2.21) and the superpotential (2.19).

\section{Two-field models}

Several of the features of the single-field model that we discussed in section 2 generalize in an interesting geometrical way to models with $N>1$ fields. We illustrate this first by considering in this section the simplest generalization, i.e., two-field models.

\subsection{Minkowski solutions}

We consider the following Kähler potential with two complex chiral fields:

$$
K=-3 \sum_{i=1}^{2} \alpha_{i} \ln \left(\phi_{i}+\phi_{i}^{\dagger}\right): \alpha_{i}>0 .
$$

with the following ansatz for the superpotential

$$
W=a \prod_{i=1}^{2} \phi_{i}^{n_{i}}
$$

Denoting the real and imaginary parts by $\phi_{i}=x_{i}+i y_{i}$, we find that the potential along the real field directions $y_{i}=0$ is given by

$$
V=\left(\sum_{i=1}^{2} \frac{\left(2 n_{i}-3 \alpha_{i}\right)^{2}}{3 \alpha_{i}}-3\right) \cdot a^{2} \cdot\left(\prod_{i}^{2} 2^{-3 \alpha_{i}} x_{i}^{2 n_{i}-3 \alpha_{i}}\right) .
$$

We see immediately that by setting

$$
\sum_{i=1}^{2} \frac{\left(2 n_{i}-3 \alpha_{i}\right)^{2}}{3 \alpha_{i}}=3
$$

we obtain a Minkowski solution, 
We observe that (3.4) describes an ellipse in the $\left(n_{1}, n_{2}\right)$ plane centred at $\left(3 \alpha_{1} / 2,3 \alpha_{2} / 2\right)$. All choices of $\left(n_{1}, n_{2}\right)$ lying on this ellipse yield a Minkowski solution. In this way, the line segment centred at $3 \alpha / 2$ in the single-field model that yielded Minkowski endpoints is generalized, and we obtain a one-dimensional continuum subspace of Minkowski solutions. We can conveniently parametrize the solutions for $n_{i}$ in (3.4) as the points on the ellipse corresponding to unit vectors $\vec{r}=\left(r_{1}, r_{2}\right)$ with $r_{1}^{2}+r_{2}^{2}=1$ :

$$
n_{i \pm}=\frac{3}{2}\left(\alpha_{i} \pm \frac{r_{i}}{\sqrt{\sum_{j=1}^{2} \frac{r_{j}^{2}}{\alpha_{j}}}}\right), \quad i=1,2
$$

The unit vector $\vec{r}$ should be located starting at the centre of the ellipse, and defines a direction on its circumference. The operation $\vec{r} \rightarrow-\vec{r}$ in equation (3.5) takes a point on the ellipse to its antipodal point, an observation we use later to construct de Sitter solutions. We note also that holomorphy requires both $n_{1}, n_{2} \geq 0$, i.e.

$$
\alpha_{i}+\frac{r_{i}}{\sqrt{\sum_{j=1}^{2} \frac{r_{j}^{2}}{\alpha_{j}}}} \geq 0, \quad i=1,2 .
$$

As in the case of the single-field model, we can move from one point on the ellipse to another point via a Kähler transformation. This is possible because the superpotential is just a monomial.

Integer solutions for the values of $n_{i}$ are also possible in the two-field case. The full set of solutions in the single-field case are valid for $n_{1 \pm}$ when $n_{2+}=n_{2-}$ (and similarly when $1 \leftrightarrow 2$ ). More generally, solutions can be found by writing

$$
\left(n_{1+}-n_{1-}\right)^{2}=\lambda_{1}\left(n_{1+}+n_{1-}\right) \quad \text { and } \quad\left(n_{2+}-n_{2-}\right)^{2}=\lambda_{2}\left(n_{2+}+n_{2-}\right),
$$

with $\lambda_{i}$ is non-negative and $\lambda_{1}+\lambda_{2}=3$. As one example out of an infinite number of solutions, choosing $\lambda_{1}=1$ and $\lambda_{2}=2$ gives $\left(n_{1+}, n_{1-}\right)=(3,1)$ and $\left(n_{2+}, n_{2-}\right)=(6,2)$.

In general, points around the ellipse yield potentials that are flat only in the real direction and, as in the single-field model, may not be stable in the imaginary directions. The masses of the imaginary component fields $y_{1}, y_{2}$ are given by

$$
m_{y_{i}}^{2}=\frac{2^{2-3\left(\alpha_{1}+\alpha_{2}\right)}}{\alpha_{i}^{2}} \cdot\left(\alpha_{i}^{2}-\frac{r_{i}^{2}}{\left(\sum_{j=1}^{2} \frac{r_{j}^{2}}{\alpha_{j}}\right)}\right) \cdot a^{2} \cdot x_{1}^{2 n_{1}-3 \alpha_{1}} x_{2}^{2 n_{2}-3 \alpha_{2}}, \quad i=1,2 .
$$

The stability requirement $m_{y_{i}}^{2} \geq 0$ for $x_{i}>0$ implies

$$
\alpha_{i}^{2}-\frac{r_{i}^{2}}{\left(\sum_{j=1}^{2} \frac{r_{j}^{2}}{\alpha_{j}}\right)} \geq 0, \quad i=1,2 .
$$

It is easy to see that if the stability conditions are satisfied then the holomorphy conditions (3.6) are satisfied. Since the left hand side of (3.9) is proportional to $n_{i_{+}} n_{i_{-}}$, points 


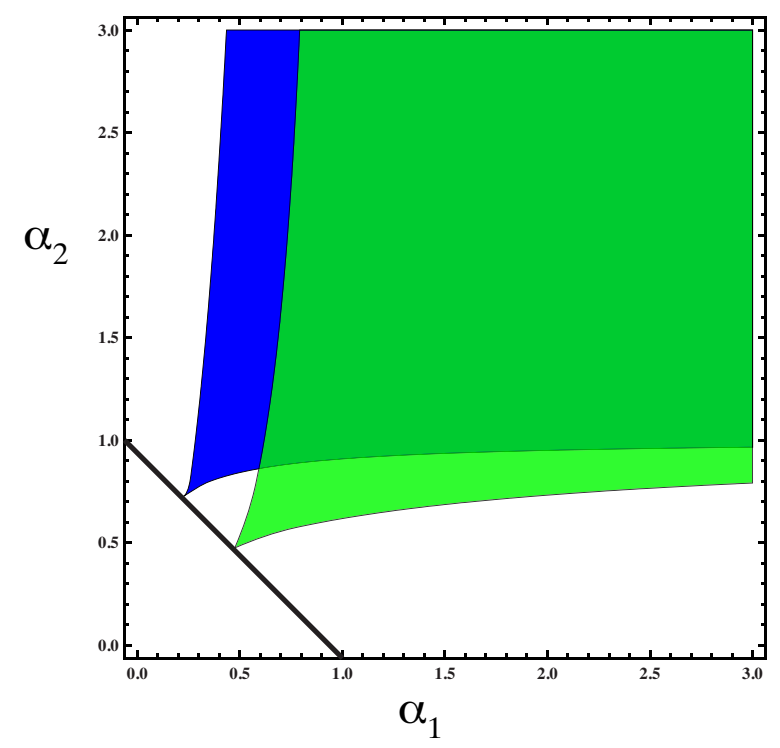

Figure 2. The shaded regions are the allowed values of $\alpha_{1}, \alpha_{2}$ for the illustrative choices $\vec{r}=$ $(1 / \sqrt{2}, 1 / \sqrt{2})$ (green) and $\vec{r}=(1 / \sqrt{10}, 3 / \sqrt{10})$ (blue). There are kinks located at $\left(\alpha_{1}, \alpha_{2}\right)=$ $(1 / 2,1 / 2)$ and $\left(\alpha_{1}, \alpha_{2}\right)=(1 / 4,3 / 4)$ for the two choices of unit vectors. The black line is $\alpha_{1}+\alpha_{2}=1$.

on the ellipse that give stable Minkowski solution are those that are holomorphic so long as their antipodal points are also holomorphic.

However, given a choice of unit vector, $\vec{r}$, this condition is not satisfied for all choices of $\alpha_{i}$. We show in figure 2 the allowed domain in the $\left(\alpha_{1}, \alpha_{2}\right)$ plane for which the stability conditions (3.9) (and hence also the holomorphy conditions (3.6)) are satisfied, for two illustrative choices of the unit vector $\vec{r}$. The allowed region for $\vec{r}=(1 / \sqrt{2}, 1 / \sqrt{2})$ is shaded green and behind it (shaded blue) is the allowed region when $\vec{r}=(1 / \sqrt{10}, 3 / \sqrt{10})$. For both choices of $\vec{r}$, there is a kink in the allowed domain where it meets the line given by $\alpha_{1}+\alpha_{2}=1$. At the kink, for all choices of $\vec{r}$, the potential is completely flat and vanishes in all directions in field space. The position of the kink can be calculated by solving the stability condition along this line:

$$
\alpha_{1}=\frac{r_{1}^{2}-\sqrt{r_{1}^{2}-r_{1}^{4}}}{2 r_{1}^{2}-1}
$$

For the two examples shown in figure $2, r_{1}=1 / \sqrt{2}$ implies $\alpha_{1}=1 / 2$ at the kink, and $r_{1}=1 / \sqrt{10}$ implies $\alpha_{1}=1 / 4$. In fact, because of the sign ambiguity, there are four unit vectors for each solution, corresponding to the ambiguous signs of $r_{1}$ and $r_{2}$.

Another projection of the domain of stability is shown in figure 3, which displays the allowed regions in the $\left(\alpha_{1}, r_{1}^{2}\right)$ plane for the fixed values $\alpha_{2} / \alpha_{1}=1,2,3,5,10$, as illustrated by the curves, respectively. Each pair of curves (red, green, purple, blue and black for increasing $\alpha_{2} / \alpha_{1}$ ) corresponds to the two equalities in (3.9), and the positivity inequalities are satisfied to the right of each pair of lines for a given value of $\alpha_{2} / \alpha_{1}$. For example, when $\alpha_{2} / \alpha_{1}=1$ (shown by the solid red curves), all values of $r_{1}^{2}$ are allowed if $\alpha_{1} \geq 1$, while no values are allowed when $\alpha_{1}<1 / 2$. The point where the curves meet corresponds to the 


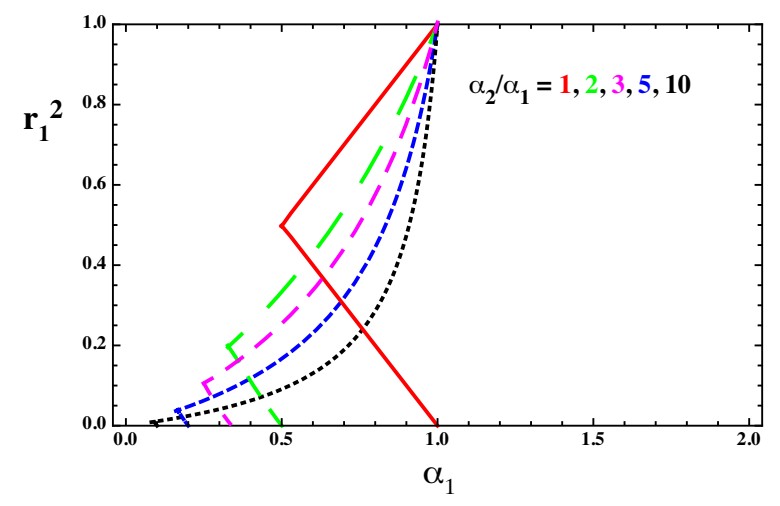

Figure 3. The allowed values of $\alpha_{1}, r_{1}^{2}$ for fixed ratios of $\alpha_{2} / \alpha_{1}=1,2,3,5,10$. The two sets of curves are derived from the two constraint equations in (3.9). The stability inequality is satisfied for points with $\alpha_{1}$ to the right of both curves of the same colour (red, green, purple, blue and black for increasing $\left.\alpha_{2} / \alpha_{1}\right)$. The point at which the two curves meet corresponds to the kink that appears in figure 2 when $\alpha_{1}+\alpha_{2}=1$.

kink when $\alpha_{1}=\alpha_{2}=1 / 2$ and $r_{1}^{2}=1 / 2$ that was seen in figure 2 where the green shaded region touches the black line. When $\alpha_{2} / \alpha_{1}=3$ (shown by medium dashed purple curves), the kink occurs when these two curves meet at $\alpha_{1}=1 / 4$ and $r_{1}^{2}=1 / 10$.

The lower ellipse (3.4) in the $\left(n_{1}, n_{2}\right)$ plane shown in figure 4 corresponds to this second example. As this corresponds to the position of the kink, only a single value of $r_{1}^{2}=1 / 10$ is allowed. The four red spots in the figure correspond to the four different vectors $\vec{r}=$ $( \pm 1 / \sqrt{10}, \pm 3 / \sqrt{10})$. These four unit vectors correspond to four different superpotentials via the relation $(3.5)$, which give $\left(n_{1}, n_{2}\right)=(3 / 4,9 / 4),(3 / 4,0),(0,9 / 4),(0,0)$. When $\left(\alpha_{1}, \alpha_{2}\right)=$ $(1 / 4,3 / 4)$, each of the four superpotentials defined by the pair $n_{i}$ yields a true Minkowski solution. However, because we are at the kink, there are no other stable solutions.

Choosing a larger value of $\alpha_{1}$ while keeping $\alpha_{2} / \alpha_{1}$ fixed would increase the allowed range in $r_{1}^{2}$ (as seen in figure 3 ) and would allow a continuum of stable Minkowski solutions along the real direction in field space. This is seen in the upper ellipse in figure 4 , where we have chosen $\alpha_{1}=1 / 2$ and $\alpha_{2}=3 / 2$. In this case, the stability constraint, which can be read off figure 3 for $\alpha_{2} / \alpha_{1}=3$ at the chosen value of $\alpha_{1}$, yields $r_{1}<1 / 2$. Unit vectors with $r_{1}<1 / 2$ correspond to arcs along the upper ellipse in figure 4 . These are further shortened by the holomorphy requirement that $n_{i} \geq 0$, and the resulting allowed solutions are shown by the red arc segments in the upper ellipse.

To summarize this discussion of Minkowski solutions in the two-field case:

1) For any generic unit vector $\vec{r}$, there is always a kink in the boundary of the allowed values of $\left(\alpha_{1}, \alpha_{2}\right)$ as shown in figure 2 , and these kink solutions always satisfy $\alpha_{1}+$ $\alpha_{2}=1$ with $\alpha_{1}$ given by (3.10). The kink solutions give a vanishing potential $V=0$ in all directions in field space.

2) For any pair $\left(\alpha_{1}, \alpha_{2}\right)$ satisfying $\alpha_{1}+\alpha_{2}=1$, there are four unit vectors that are 


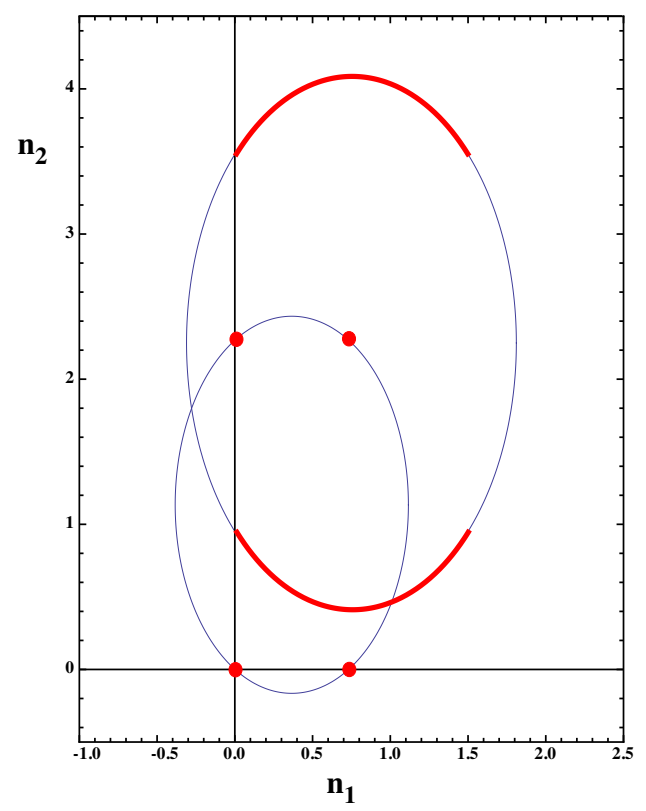

Figure 4. Minkowski solutions for $\alpha_{1}=1 / 4, \alpha_{2}=3 / 4$ (lower ellipse) and $\alpha_{1}=1 / 2, \alpha_{2}=3 / 2$ (upper ellipse). In the former case only the four red points corresponding to $\vec{r}=( \pm 1 / \sqrt{10}, \pm 3 / \sqrt{10})$ are allowed, whereas in the latter case the red arc segments correspond to allowed solutions.

determined by inverting (3.10), namely

$$
r_{1}= \pm \frac{\alpha_{1}}{\sqrt{1-2 \alpha_{1}+2 \alpha_{1}^{2}}} .
$$

The four values of the $n_{i}$ that correspond to these choices are $\left(n_{1}, n_{2}\right)=$ $(0,0),\left(3 \alpha_{1}, 0\right),\left(0,3 \alpha_{2}\right),\left(3 \alpha_{1}, 3 \alpha_{2}\right)$.

3) For $\alpha_{1}+\alpha_{2}>1$, a continuum of stable Minkowski solutions exist and, when $\alpha_{1} \geq 1$ with $\alpha_{2} / \alpha_{1} \geq 1$, the entire ellipse (that is, all unit vectors $\vec{r}$ ) yield stable Minkowski solutions in the real directions of field space.

4) The holomorphy conditions (3.6) are satisfied automatically if the stability conditions (3.9) are satisfied.

5) There is an infinite set of Minkowski solutions with positive integer powers of the fields in the superpotential.

\subsection{De Sitter solutions}

We recall that in the single-field model we were able to construct a de Sitter solution by combining the two superpotentials corresponding to Minkowski solutions that can be visualized as opposite ends of a line segment. In the two-field model, we have a continuum of superpotentials that give Minkowski solutions, which are described by an ellipse (3.4). In this case it is possible to to construct new de Sitter solutions by combining superpotentials 
corresponding to antipodal points on the ellipse (3.4). For example, consider the following combined superpotential:

$$
W=a\left(\phi_{1}^{n_{1+}} \phi_{2}^{n_{2+}}-\phi_{1}^{n_{1-}} \phi_{2}^{n_{2-}}\right) .
$$

It is easy to see that the scalar potential in the real field direction is a de Sitter solution:

$$
V=3 \cdot 2^{2-3 \alpha_{1}-3 \alpha_{2}} \cdot a^{2}
$$

in this case.

For the example described by the lower ellipse in figure 4, one example of a de Sitter solution is found by taking antipodal points corresponding to the red spots. When $\vec{r}=(1 / \sqrt{10}, 3 / \sqrt{10})$, we have $W=a\left(\phi_{1}^{3 / 4} \phi_{2}^{9 / 4}-1\right)$, which is the unique solution with a holomorphic superpotential that results in a flat de Sitter potential in the real direction. However, as we discuss further below, this solution is actually not stable.

As an alternative example, we consider a two-field model with $\alpha_{1}=1$ and $\alpha_{2}=2$. The Minkowski solutions in this case are described by the ellipse (3.4) in $\left(n_{1}, n_{2}\right)$ space shown in figure 5 , whose centre is at $(3 / 2,3)$. In this case, the entire ellipse can be used to construct de Sitter solutions, as all possible unit vectors $\vec{r}$ are allowed since $\alpha_{1}>1$ (see figure 3). As in the previous example, we can use antipodal points to construct de Sitter solutions, as illustrated in figure 5. One such pair of antipodal points is $(3,3),(3,0)$, corresponding to $\vec{r}=(1,0)$, indicated by the horizontal orange line in figure 5 . The corresponding superpotential is

$$
W=a^{2}\left(\phi_{1}^{3} \phi_{2}^{3}-\phi_{2}^{3}\right)
$$

so that the fields appear in the superpotential with positive integer powers. This example yields a de Sitter potential with the potential value

$$
V=3 \cdot 2^{-7} \cdot a^{2}
$$

along the real field directions. A continuum of de Sitter solutions for real field values are possible for different choices of $\vec{r}$, e.g., the choice indicated in figure 5 by the blue line, all with the potential given by eq. (3.15).

\subsection{Stability analysis}

As in the single-field case, the de Sitter solutions of the two-field model require modification in order to be stable. Stable solutions can easily be found by deforming the Kähler potential to include stabilizing quartic terms:

$$
K=-3 \sum_{i=1}^{2} \alpha_{i} \ln \left(\phi_{i}+\phi_{i}^{\dagger}+b_{i}\left(\phi_{i}-\phi_{i}^{\dagger}\right)^{4}\right): b_{i}>0 .
$$

With this modification the potential along real field directions is still given by equation (3.13). To prove the stability of the two-field de Sitter solution with the quartic modification of the Kähler potential, we calculate the Hessian matrix $\partial^{2} V / \partial y_{i} \partial y_{j}: i, j=1,2$ 


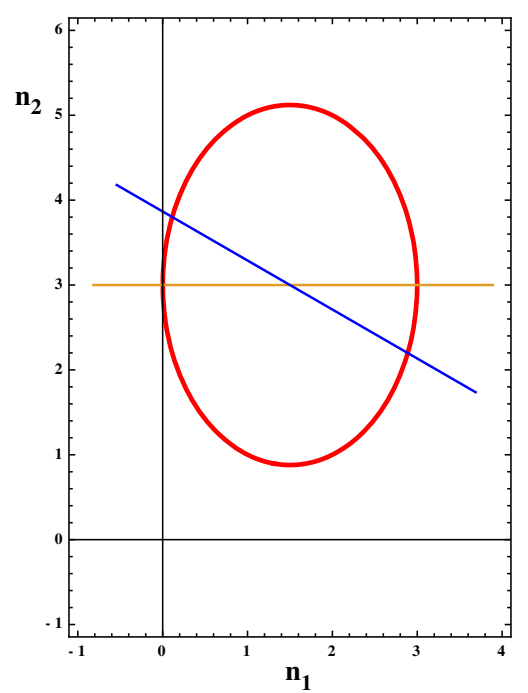

Figure 5. The Minkowski solutions for $\alpha_{1}=1$ and $\alpha_{2}=2$ are described by an ellipse in $\left(n_{1}, n_{2}\right)$ space. Lines passing through the center of the ellipse connect antipodal points, as illustrated with two examples.

along the real field directions, and demand that it be positive semi-definite. The Hessian matrix along the real field directions is of the form

$$
a^{2}\left(\frac{3.2^{1-3 \alpha_{1}-3 \alpha_{2}}}{\alpha_{2} r_{1}^{2}+\alpha_{1} r_{2}^{2}}\right)\left[\begin{array}{cc}
x_{1}^{-2} A_{1} & x_{1}^{-1} x_{2}^{-1} B \\
x_{1}^{-1} x_{2}^{-1} B & x_{2}^{-2} A_{2}
\end{array}\right],
$$

where

$$
\begin{aligned}
A_{1} & =w^{-1}\left(\alpha_{1}^{2} r_{2}^{2}\left(1+4 w+w^{2}\right)+\alpha_{1} \alpha_{2} r_{1}^{2}(1-w)^{2}+\alpha_{2} r_{1}^{2}\left(96 b_{1} x_{1}^{3}-1\right)(1+w)^{2}\right), \\
A_{2} & =w^{-1}\left(\alpha_{2}^{2} r_{1}^{2}\left(1+4 w+w^{2}\right)+\alpha_{1} \alpha_{2} r_{2}^{2}(1-w)^{2}+\alpha_{1} r_{2}^{2}\left(96 b_{2} x_{2}^{3}-1\right)(1+w)^{2}\right), \\
B & =-6 \alpha_{1} \alpha_{2} r_{1} r_{2},
\end{aligned}
$$

we have defined

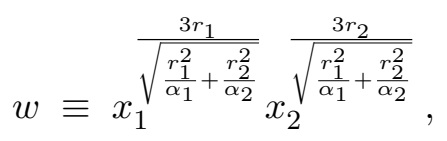

and the Hessian matrix is positive semi-definite if the condition

$$
\mathcal{H} \equiv A_{1} A_{2} \geq B^{2}
$$

is satisfied.

The stability condition (3.22) for generic $\alpha_{1}, \alpha_{2}, b_{1}, b_{2}$ and $\vec{r}$ is

$$
\begin{aligned}
& \left(\alpha_{1}^{2} r_{2}^{2}\left(1+4 w+w^{2}\right)+\alpha_{1} \alpha_{2} r_{1}^{2}(1-w)^{2}+\alpha_{2} r_{1}^{2}\left(96 b_{1} x_{1}^{3}-1\right)(1+w)^{2}\right) \\
& \quad \times\left(\alpha_{2}^{2} r_{1}^{2}\left(1+4 w+w^{2}\right)+\alpha_{1} \alpha_{2} r_{2}^{2}(1-w)^{2}+\alpha_{1} r_{2}^{2}\left(96 b_{2} \frac{w^{\frac{1}{r_{2}} \sqrt{\frac{r_{1}^{2}}{\alpha_{1}}+\frac{r_{2}^{2}}{\alpha_{2}}}}}{x_{1}^{\left(3 r_{1} / r_{2}\right)}}-1\right)(1+w)^{2}\right) \\
& \quad-36 \alpha_{1}^{2} \alpha_{2}^{2} r_{1}^{2} r_{2}^{2} w^{2} \geq 0 .
\end{aligned}
$$


A general stability analysis is intractable, so we have considered the simplified case: $\alpha_{1}=$ $\alpha_{2} \equiv \alpha$ and $\vec{r}=(1 / \sqrt{2}, 1 / \sqrt{2})$, for which the positivity condition (3.22) becomes

$$
\begin{aligned}
& \left(2 \alpha\left(1+w+w^{2}\right)+\left(96 b_{1} x_{1}^{3}-1\right)(1+w)^{2}\right) \\
& \quad \times\left(2 \alpha\left(1+w+w^{2}\right)+\left(96 b_{2} x_{2}^{3}-1\right)(1+w)^{2}\right) \geq 36 \alpha^{2} w^{2} .
\end{aligned}
$$

Eliminating $x_{2}$ in favour of $x_{1}$ and $w$ via equation (3.21), this inequality becomes

$$
\begin{aligned}
& \left(2 \alpha\left(1+w+w^{2}\right)+\left(96 b_{1} x_{1}^{3}-1\right)(1+w)^{2}\right) \\
& \quad \times\left(2 \alpha\left(1+w+w^{2}\right)+\left(96 b_{2} \frac{w^{\sqrt{2 / \alpha}}}{x_{1}^{3}}-1\right)(1+w)^{2}\right)-36 \alpha^{2} w^{2} \geq 0 .
\end{aligned}
$$

We note that $\left(96 b_{1} x_{1}^{3}-1\right)$ dominates for $x_{1} \gg 1$ and $\left(96 b_{2} \frac{w^{\sqrt{2 / \alpha}}}{x_{1}^{3}}-1\right)$ dominates for $x_{1} \ll 1$, implying that there is an extremum for some intermediate value of $x_{1}$. This occurs at $x_{1}=\left(b_{2} / b_{1}\right)^{1 / 6} w^{1 /(3 \sqrt{2 \alpha})}$, and is a global extremum. Whether it is a maximum or a minimum depends on the sign of $2 \alpha\left(1+w+w^{2}\right)-(1+w)^{2}$, and it is non-negative for

$$
\alpha \geq \frac{2}{3}
$$

This is a necessary condition for the inequality (3.25) to be satisfied. We have not explored the full range of possible values of $b_{1}$ and $b_{2}$ when $\alpha_{1}=\alpha_{2}=\alpha$, but have checked that the stability condition (3.25) is always satisfied if $b_{1}=b_{2}=1$ and $\alpha \geq 2 / 3$, irrespective of the value of $w$. We have also found that when $\alpha_{1} \neq \alpha_{2}$ the sum $\alpha_{1}+\alpha_{2} \geq 4 / 3$.

We have also considered the case $\vec{r}=(0,1)$ with $b_{1}=b_{2}=1$. The inequality (3.23) reduces in this case to

$$
\alpha_{2}(1-w)^{2}+(1+w)^{2}\left(96 w^{1 / \sqrt{\alpha_{2}}}-1\right) \geq 0,
$$

which is always satisfied for $\alpha_{2} \geq 1$. It is easy to check that the same is true for the case $\vec{r}=(1,0)$. Based on these cases and the previous example with $\vec{r}=(1 / \sqrt{2}, 1 / \sqrt{2})$, we expect that there are generic stable solutions for a range of $\vec{r}$ in the first and third quadrants where $r_{1} / r_{2}>0$. However, the situation is different when $r_{1} / r_{2}<0$. We find that the inequality (3.22) cannot be satisfied for $\vec{r}=(-1 / \sqrt{2}, 1 / \sqrt{2})$ and $b_{1}=b_{2}=1$, so there are no stable de Sitter solutions, and we expect the same to be the case for other choices of $\vec{r}$ in the second or fourth quadrant.

In summary, we have established the existence of stable de Sitter solutions only when $\vec{r}$ is in either first or third quadrant.

\section{$4 \quad$ N-field models}

Finally, we generalize the above set of examples to models with multiple fields $N>2$.

\subsection{Minkowski Solutions}

The natural generalization of the Kähler potential in (3.1) is simply a sum of $N$ similar terms:

$$
K=-3 \sum_{i=1}^{N} \alpha_{i} \ln \left(\phi_{i}+\phi_{i}^{\dagger}\right)
$$


Similarly, we adopt the following ansatz for the superpotential:

$$
W=a \prod_{i=1}^{N} \phi_{i}^{n_{i}}
$$

in which case the potential along the real field directions $x_{i}$ is

$$
V=\left(\sum_{i=1}^{N} \frac{\left(2 n_{i}-3 \alpha_{i}\right)^{2}}{3 \alpha_{i}}-3\right) \cdot a^{2} \cdot\left(\prod_{i}^{N} 2^{-3 \alpha_{i}} x_{i}^{2 n_{i}-3 \alpha_{i}}\right) .
$$

We can obtain Minkowski solutions along the real field directions by setting

$$
\sum_{i=1}^{N} \frac{\left(2 n_{i}-3 \alpha_{i}\right)^{2}}{3 \alpha_{i}}=3
$$

which describes an ellipsoid in $\left(n_{1}, \ldots, n_{N}\right)$ space whose centre is at $\left(3 \alpha_{1} / 2, \ldots, 3 \alpha_{N} / 2\right)$. Once again we find a continuum of Minkowski solutions. The points on the ellipsoid can be parametrized conveniently using an $N$-dimensional unit vector $\vec{r}$ :

$$
n_{i}=\frac{3}{2}\left(\alpha_{i}+\frac{r_{i}}{\sqrt{\sum_{j=1}^{N} \frac{r_{j}^{2}}{\alpha_{j}}}}\right) \quad i=1, \ldots, N ; \quad r_{1}^{2}+\ldots+r_{N}^{2}=1,
$$

where the unit vector $\vec{r}$ is to be considered as anchored at the centre of the ellipsoid. To ensure holomorphy of the superpotential we need $n_{i} \geq 0$, and the masses of the imaginary field components $y_{i}$ are given by

$$
m_{y_{i}}^{2}=\frac{2^{2-3\left(\sum \alpha_{i}\right)}}{\alpha_{i}^{2}} \cdot\left(\alpha_{i}^{2}-\frac{r_{i}^{2}}{\left(\sum_{j=1}^{N} \frac{r_{j}^{2}}{\alpha_{j}}\right)}\right) \cdot a^{2} \cdot \prod_{j=1}^{N} x_{j}^{2 n_{j}-3 \alpha_{j}}, \quad i=1, \ldots, N .
$$

For stability, we impose conditions similar to (3.9), namely:

$$
\alpha_{i}^{2}-\frac{r_{i}^{2}}{\left(\sum_{j=1}^{2} \frac{r_{j}^{2}}{\alpha_{j}}\right)} \geq 0 \quad i=1, \ldots, N
$$

As in two-field models, ensuring these stability conditions are satisfied implies that the holomorphy conditions are also satisfied. For a given unit vector $\vec{r}$, one can ask what values of $\alpha_{1}, \ldots, \alpha_{N}$ satisfy the stability conditions. We find a multidimensional region analogous to that in figure 2 , with a vertex that satisfies $\alpha_{1}+\ldots+\alpha_{N}=1$. We show in figure 6 the allowed region of $\alpha_{1}, \alpha_{2}$ and $\alpha_{3}$ for a three-field model with $\vec{r}=(1 / \sqrt{3}, 1 / \sqrt{3}, 1 / \sqrt{3})$.

The vertex is a special solution that corresponds to $V=0$ in both the real and imaginary field directions. When the sign of one of the components of $\vec{r}$ is changed, the region in $\alpha_{1}, \ldots, \alpha_{N}$ space that satisfies (4.7) remains the same. Therefore, there are 


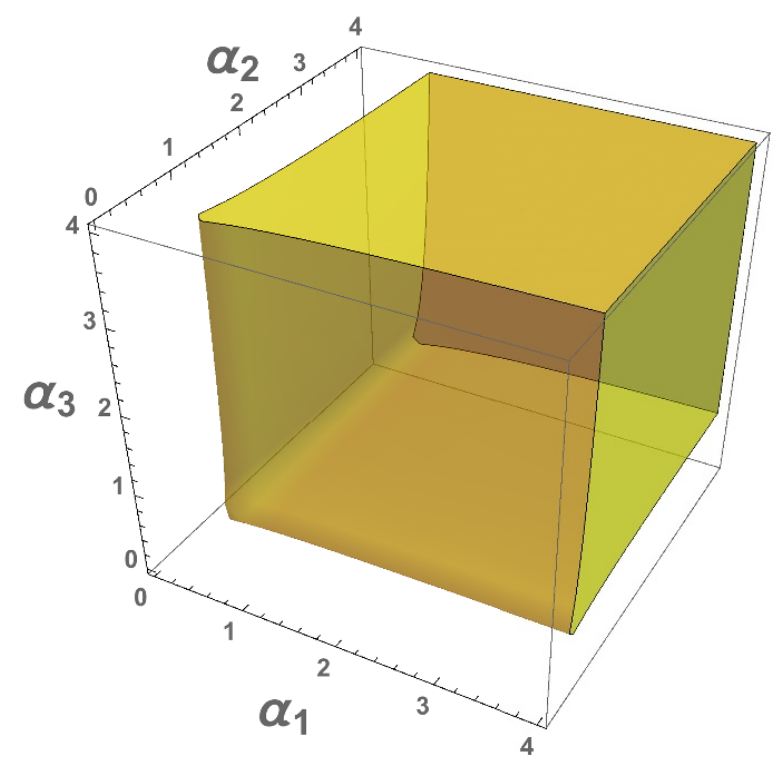

Figure 6. Allowed values of $\alpha_{1}, \alpha_{2}, \alpha_{3}$ for a three-field model with $\vec{r}=(1 / \sqrt{3}, 1 / \sqrt{3}, 1 / \sqrt{3})$.

$2^{N}$ unit vectors, each differing only in the sign of the components, that have the same vertex solution.

The above observations on the vertex solution can be summarized as follows. When

$$
\sum_{i=1}^{N} \alpha_{i}=1
$$

there are $2^{N}$ superpotentials of the form

$$
W=a \phi_{i_{1}}^{3 \alpha_{i_{1}}} \ldots \phi_{i_{n}}^{3 \alpha_{i_{n}}}
$$

where $\left\{i_{1}, \ldots, i_{n}\right\}(n \leq N)$ is a subset of $\{1,2, \ldots, N\}$, that all give $V=0$ in both the real and imaginary field directions.

\subsection{De Sitter solutions}

Finally we discuss de Sitter solutions in $N$-field models. Here the Kähler potential is again given by

$$
K=-3 \sum_{i=1}^{N} \alpha_{i} \ln \left(\phi_{i}+\phi_{i}^{\dagger}\right),
$$

and, as in the two-field case, the superpotential may be constructed from two antipodal points of the ellipse (4.4):

$$
W=a\left(\prod_{i=1}^{N} \phi_{i}^{n_{i+}}-\prod_{i=1}^{N} \phi_{i}^{n_{i-}}\right)
$$




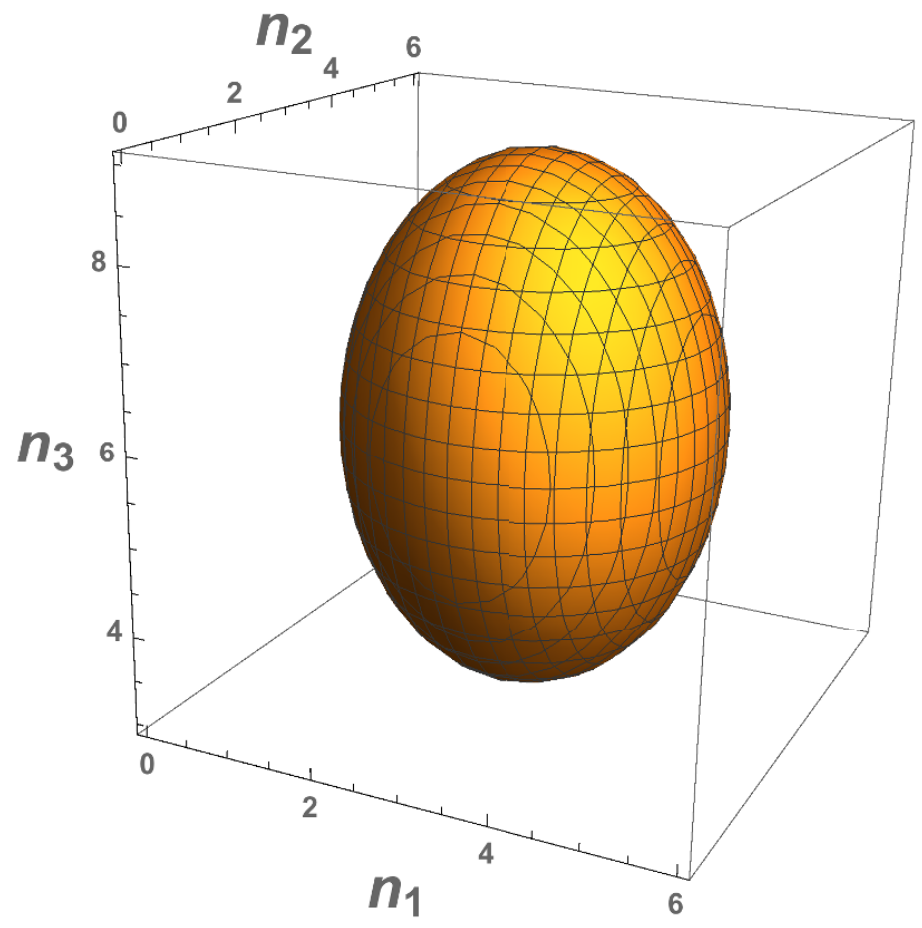

Figure 7. Minkowski solutions for the three-field model with $\alpha_{1}=2, \alpha_{2}=2$ and $\alpha_{3}=4$.

where the exponents are given by

$$
n_{i \pm}=\frac{3}{2}\left(\alpha_{i} \pm \frac{r_{i}}{\sqrt{\sum_{j=1}^{N} \frac{r_{j}^{2}}{\alpha_{j}}}}\right) \quad i=1, \ldots, N ; \quad r_{1}^{2}+\ldots+r_{N}^{2}=1,
$$

and the potential along the real field directions is then

$$
V=3 \cdot 2^{\left(2-3 \sum_{i=1}^{N} \alpha_{i}\right)} \cdot a^{2} .
$$

We use a simple three-field model with $\alpha_{1}=2, \alpha_{2}=2$ and $\alpha_{3}=4$ for illustration. The Minkowski solutions are described by an ellipsoid in $\left(n_{1}, n_{2}, n_{3}\right)$ space centred at $(3,3,6)$, which is shown in figure 7 .

To construct de Sitter solutions for this model, we choose the antipodal points $(3,3,9),(3,3,3)$ corresponding to the unit vector $\vec{r}=(0,0,1)$, which yield the superpotential:

$$
W=a\left(\phi_{1}^{3} \phi_{2}^{3} \phi_{3}^{9}-\phi_{1}^{3} \phi_{2}^{3} \phi_{3}^{3}\right) .
$$

This yields a de Sitter potential along the real field directions with potential

$$
V=3 \cdot 2^{-16} \cdot a^{2} .
$$

\subsection{Stability analysis}

The stability analysis of the de Sitter solution in the $N$-field model is difficult, as it requires finding the eigenvalues of an $N \times N$ matrix. However, as in the two-field model, we do not 
expect the solution to be stable unless the Kähler potential is deformed, e.g., to

$$
K=-3 \sum_{i=1}^{N} \alpha_{i} \ln \left(\phi_{i}+\phi_{i}^{\dagger}+b_{i}\left(\phi_{i}-\phi_{i}^{\dagger}\right)^{4}\right) .
$$

With this modification, for any given unit vector $\vec{r}$ there should exist a region in $\left(\alpha_{1}, \ldots, \alpha_{N}\right)$ space where the de Sitter solution is stable.

To demonstrate this in a specific three-field example, we consider the model with three chiral fields $S, T, U$ that was considered in [42]. This model is defined by the following Kähler potential and superpotential:

$$
\begin{aligned}
& K=-\ln \left(S+S^{\dagger}\right)-3 \ln \left(T+T^{\dagger}\right)-3 \ln \left(U+U^{\dagger}\right), \\
& W=W(S, T, U) .
\end{aligned}
$$

This model is of particular interest as it arises in the compactification of Type IIB string theory on $T^{6} / \mathbb{Z}_{2} \times \mathbb{Z}_{2}$. Then the three chiral fields are the axiodilaton $S$, a volume modulus $T$ and a complex structure modulus $U$. One expects that the perturbative contribution to the superpotential should be a polynomial and that the non-perturbative contribution would have a decaying exponential form. For our analysis we assume that the powers of the fields in the superpotential could also be fractional. In our notations, this $S T U$ model has $\alpha_{1}=1 / 3, \alpha_{2}=1$ and $\alpha_{3}=1$.

We first construct a Minkowski solution. We can use the stability conditions to find a unit vector $\vec{r}$ and construct an appropriate superpotential. One such unit vector is $\vec{r}=(0,1,0)$. This leads to a superpotential of the form

$$
W=a S^{1 / 2} T^{3} U^{3 / 2},
$$

which gives a stable Minkowski solution $V=0$ along real field directions. ${ }^{6}$

In order to construct de Sitter solutions we add stabilization terms to the Kähler potential:

$$
K=-\ln \left(S+S^{\dagger}+b_{S}\left(S-S^{\dagger}\right)^{4}\right)-3 \ln \left(T+T^{\dagger}+b_{T}\left(T-T^{\dagger}\right)^{4}\right)-3 \ln \left(U+U^{\dagger}+b_{U}\left(U-U^{\dagger}\right)^{4}\right) .
$$

As discussed above, we use antipodal points to construct the superpotential, choosing $\vec{r}=(0, \pm 1,0)$, in which case:

$$
W=a S^{1 / 2} U^{3 / 2}\left(T^{3}-1\right) .
$$

With this we get a de Sitter solution along the real field directions with potential

$$
V=\frac{3}{32} \cdot a^{2} .
$$

In order to check whether the de Sitter solution is stable for the antipodal points that we have chosen, we calculate the Hessian matrix along the real field directions to verify that the eigenvalues are non-negative. Defining

$$
S=s+i y_{1}, \quad T=t+i y_{2}, \quad U=u+i y_{3},
$$

\footnotetext{
${ }^{6}$ We mention in passing that the $S T U$ model does not admit any superpotential with only integer powers, for either Minkowski or de Sitter solutions.
} 
we calculate the Hessian matrix $\partial^{2} V / \partial y_{i} \partial y_{j}: i, j=1,2,3$ along the real field directions, finding

$$
\left[\begin{array}{ccc}
\frac{a^{2}\left(1+4 t^{3}+t^{6}\right)}{64 s^{2} t^{3}} & 0 & 0 \\
0 & \frac{-3 a^{2}+72 a^{2} b_{T}\left(1+t^{3}\right)^{2}}{16 t^{2}} & 0 \\
0 & 0 & \frac{3 a^{2}\left(1+4 t^{3}+t^{6}\right)}{64 t^{3} u^{2}}
\end{array}\right]
$$

We see that the Hessian matrix is diagonal, so the eigenvalues are simply the diagonal entries. For the Hessian matrix to be positive semi-definite we need

$$
-3 a^{2}+72 a^{2} b_{T}\left(1+t^{3}\right)^{2} \geq 0
$$

which is independent of $b_{S}$ and $b_{U}$. Therefore, we simply need

$$
b_{T} \geq \frac{1}{24},
$$

with no restriction on $b_{S}$ and $b_{U}$.

\section{Conclusion and outlook}

Generalizing previous discussions of de Sitter solutions in single-field no-scale models [3, $36,40]$, in this paper we have discussed de Sitter solutions in multi-field no-scale models as may appear in realistic string compactifications with multiple moduli.

As a preliminary, we showed that the space of Minkowski vacua in multi-field no-scale models is characterized by the surface of an ellipsoid. The parameters in these models are the coefficients $\left(\alpha_{1}, \ldots, \alpha_{N}\right)$ in the generalized no-scale Kähler potential and a unit vector $\vec{r}$ that selects a particular pair of antipodal points on this ellispoid whose center is located at $\left(3 \alpha_{1} / 2, \ldots, 3 \alpha_{N} / 2\right)$. Requiring the stability of Minkowski solutions for a fixed $\vec{r}$ leads us to a region in $\left(\alpha_{1}, \ldots, \alpha_{N}\right)$ space with a vertex that is a special point where $\sum_{i=1}^{N} \alpha_{i}=1$. Such points describe Minkowski vacua with potentials that are flat in both the real and imaginary field directions. In this way we constructed $2^{N}$ monomial (in each field) superpotentials for models with $\sum_{i=1}^{N} \alpha_{i}=1$ that yield acceptable Minkowski vacua. The exponent of each monomial is determined by the coefficients $\alpha_{i}$ and the vectors, $r_{i}$.

We then constructed de Sitter solutions by combining the superpotentials at antipodal points, generalizing a construction given originally in the single-field case in [3]. These de Sitter solutions are unstable if the simple no-scale Kähler potential is used, and require stabilization. We showed that modifying the Kähler potential with a quartic term stabilizes a specific two-field model with $\alpha_{1}=\alpha_{2}=\alpha$ and $\vec{r}=(1 / \sqrt{2}, 1 / \sqrt{2})$ for $\alpha \geq 2 / 3$, and we expect the stability to hold for other generic $\vec{r}$ for suitable ranges of $\alpha_{1}, \alpha_{2}$. We also expect that similar stable de Sitter solutions exist for $N$-field models under certain conditions, as demonstrated explicitly in a specific three-field model motivated by the compactification of Type IIB string theory [42].

We note that satisfying the stability requirement also ensures that the superpotential is holomorphic in the Minkowski case, i.e., contains only positive powers of the chiral fields, whereas this is not necessarily true in the de Sitter case. It is easy to find infinite discrete 
series of models for which these powers are integral, and we have provided a number of illustrative single- and multi-field examples.

As noted in the Introduction, it is currently debated whether string theory admits de Sitter solutions [17-29]. If this were not the case, measurements of the accelerating expansion of the Universe [7-9] and the continuing success of cosmological inflation [10-16] would suggest that our Universe lies in the swampland. Our working hypothesis is that this is not the case, and that deeper understanding of string theory will reveal how it can accommodate de Sitter solutions. Since no-scale supergravity is the appropriate framework for discussing cosmology at scales hierarchically smaller than the string scale, assuming also that $N=1$ supersymmetry holds down to energies $\ll m_{\text {Planck }}$, the explorations in this paper may provide a helpful guide to the structure of the low-energy effective field theories of de Sitter string solutions. As such, they may even provide some useful signposts towards the construction of such solutions.

\section{Acknowledgments}

B.N. thanks William Linch III and Daniel Butter for useful discussions. The work of J.E. was supported in part by STFC (U.K.) via the research grant ST/L000258/1 and in part by the Estonian Research Council via a Mobilitas Pluss grant. The work of B.N. was supported by the Mitchell/Heep Chair in High Energy Physics, Texas A\&M University. The work of D.V.N. was supported in part by the DOE grant DE-FG02-13ER42020 and in part by the Alexander S. Onassis Public Benefit Foundation. The work of K.A.O. was supported in part by DOE grant DE-SC0011842 at the University of Minnesota.

Open Access. This article is distributed under the terms of the Creative Commons Attribution License (CC-BY 4.0), which permits any use, distribution and reproduction in any medium, provided the original author(s) and source are credited.

\section{References}

[1] E. Cremmer, S. Ferrara, C. Kounnas and D.V. Nanopoulos, Naturally vanishing cosmological constant in $N=1$ supergravity, Phys. Lett. 133B (1983) 61 [INSPIRE].

[2] J.R. Ellis, A.B. Lahanas, D.V. Nanopoulos and K. Tamvakis, No-scale supersymmetric standard model, Phys. Lett. 134B (1984) 429 [INSPIRE].

[3] J.R. Ellis, C. Kounnas and D.V. Nanopoulos, Phenomenological SU $(1,1)$ supergravity, Nucl. Phys. B 241 (1984) 406 [INSPIRE].

[4] J.R. Ellis, C. Kounnas and D.V. Nanopoulos, No scale supersymmetric guts, Nucl. Phys. B 247 (1984) 373 [INSPIRE].

[5] A.B. Lahanas and D.V. Nanopoulos, The road to no scale supergravity, Phys. Rept. 145 (1987) 1 [INSPIRE].

[6] E. Witten, Dimensional reduction of superstring models, Phys. Lett. 155B (1985) 151 [INSPIRE]. 
[7] Supernova Search Team collaboration, A.G. Riess et al., Observational evidence from supernovae for an accelerating universe and a cosmological constant, Astron. J. 116 (1998) 1009 [astro-ph/9805201] [INSPIRE].

[8] Supernova Cosmology Project collaboration, S. Perlmutter et al., Measurements of $\Omega$ and $\Lambda$ from 42 high redshift supernovae, Astrophys. J. 517 (1999) 565 [astro-ph/9812133] [INSPIRE].

[9] Planck collaboration, N. Aghanim et al., Planck 2018 results. VI. Cosmological parameters, arXiv: 1807.06209 [INSPIRE].

[10] K.A. Olive, Inflation, Phys. Rept. 190 (1990) 307 [INSPIRE].

[11] A.D. Linde, Particle physics and inflationary cosmology, Harwood, Chur Switzerland (1990).

[12] D.H. Lyth and A. Riotto, Particle physics models of inflation and the cosmological density perturbation, Phys. Rept. 314 (1999) 1 [hep-ph/9807278] [INSPIRE].

[13] J. Martin, C. Ringeval and V. Vennin, Encyclopædia inflationaris, Phys. Dark Univ. 5-6 (2014) 75 [arXiv: 1303.3787] [INSPIRE].

[14] J. Martin, C. Ringeval, R. Trotta and V. Vennin, The best inflationary models after Planck, JCAP 03 (2014) 039 [arXiv: 1312.3529] [INSPIRE].

[15] J. Martin, The observational status of cosmic inflation after Planck, Astrophys. Space Sci. Proc. 45 (2016) 41 [arXiv: 1502.05733] [INSPIRE].

[16] Planck collaboration, Y. Akrami et al., Planck 2018 results. X. Constraints on inflation, arXiv: 1807.06211 [INSPIRE].

[17] D.G. Boulware and S. Deser, Effective gravity theories with dilatons, Phys. Lett. B 175 (1986) 409 [INSPIRE].

[18] S. Kalara and K.A. Olive, Difficulties for field theoretical inflation in string models, Phys. Lett. B 218 (1989) 148 [INSPIRE].

[19] S. Kalara, C. Kounnas and K.A. Olive, Gravitational equations of motion at the string scale, Phys. Lett. B 215 (1988) 265 [inSPIRE].

[20] S. Kachru, R. Kallosh, A.D. Linde and S.P. Trivedi, De Sitter vacua in string theory, Phys. Rev. D 68 (2003) 046005 [hep-th/0301240] [INSPIRE].

[21] B. Michel, E. Mintun, J. Polchinski, A. Puhm and P. Saad, Remarks on brane and antibrane dynamics, JHEP 09 (2015) 021 [arXiv: 1412.5702] [inSPIRE].

[22] J. Polchinski, Brane/antibrane dynamics and KKLT stability, arXiv:1509.05710 [INSPIRE].

[23] U.H. Danielsson and T. Van Riet, What if string theory has no de Sitter vacua?, Int. J. Mod. Phys. D 27 (2018) 1830007 [arXiv: 1804.01120] [INSPIRE].

[24] G. Obied, H. Ooguri, L. Spodyneiko and C. Vafa, De Sitter space and the swampland, arXiv: 1806.08362 [INSPIRE].

[25] J.P. Conlon, The de Sitter swampland conjecture and supersymmetric AdS vacua, Int. J. Mod. Phys. A 33 (2018) 1850178 [arXiv:1808. 05040] [INSPIRE].

[26] R. Kallosh and T. Wrase, dS supergravity from 10d, Fortsch. Phys. 2018 (2018) 1800071 [arXiv: 1808.09427] [INSPIRE].

[27] R. Kallosh, A. Linde, E. McDonough and M. Scalisi, de Sitter vacua with a nilpotent superfield, arXiv: 1808.09428 [INSPIRE]. 
[28] Y. Akrami, R. Kallosh, A. Linde and V. Vardanyan, The landscape, the swampland and the era of precision cosmology, arXiv:1808.09440 [INSPIRE].

[29] S. Kachru and S.P. Trivedi, A comment on effective field theories of flux vacua, arXiv: 1808.08971 [INSPIRE].

[30] M. Gomez-Reino and C.A. Scrucca, Locally stable non-supersymmetric Minkowski vacua in supergravity, JHEP 05 (2006) 015 [hep-th/0602246] [INSPIRE].

[31] L. Covi et al., De Sitter vacua in no-scale supergravities and Calabi-Yau string models, JHEP 06 (2008) 057 [arXiv: 0804.1073] [INSPIRE].

[32] L. Covi et al., Constructing de Sitter vacua in no-scale string models without uplifting, JHEP 03 (2009) 146 [arXiv: 0812.3864] [INSPIRE].

[33] C. Kounnas, D. Lüst and N. Toumbas, $R^{2}$ inflation from scale invariant supergravity and anomaly free superstrings with fluxes, Fortsch. Phys. 63 (2015) 12 [arXiv:1409.7076] [INSPIRE].

[34] M.C.D. Marsh, B. Vercnocke and T. Wrase, Decoupling and de Sitter vacua in approximate no-scale supergravities, JHEP 05 (2015) 081 [arXiv:1411.6625] [INSPIRE].

[35] D. Gallego, M.C.D. Marsh, B. Vercnocke and T. Wrase, A new class of de Sitter vacua in type IIB large volume compactifications, JHEP 10 (2017) 193 [arXiv:1707.01095] [INSPIRE].

[36] D. Roest and M. Scalisi, Cosmological attractors from $\alpha$-scale supergravity, Phys. Rev. D 92 (2015) 043525 [arXiv: 1503. 07909] [inSPIRE].

[37] J. Ellis, D.V. Nanopoulos and K.A. Olive, Starobinsky-like inflationary models as avatars of no-scale supergravity, JCAP 10 (2013) 009 [arXiv:1307.3537] [INSPIRE].

[38] R. Kallosh, A. Linde and D. Roest, Superconformal inflationary $\alpha$-attractors, JHEP 11 (2013) 198 [arXiv: 1311.0472] [inSPIRE].

[39] R. Kallosh, A. Linde and D. Roest, Large field inflation and double $\alpha$-attractors, JHEP 08 (2014) 052 [arXiv:1405.3646] [INSPIRE].

[40] J. Ellis, D.V. Nanopoulos and K.A. Olive, From $R^{2}$ gravity to no-scale supergravity, Phys. Rev. D 97 (2018) 043530 [arXiv: 1711.11051] [INSPIRE].

[41] J.R. Ellis, C. Kounnas and D.V. Nanopoulos, No scale supergravity models with a Planck mass gravitino, Phys. Lett. 143B (1984) 410 [INSPIRE].

[42] R. Kallosh, A. Linde, B. Vercnocke and T. Wrase, Analytic classes of metastable de Sitter vacua, JHEP 10 (2014) 011 [arXiv:1406.4866] [INSPIRE]. 